\title{
Contribution of transposable elements in the plant's genome
}

\begin{abstract}
Plants maintain extensive growth flexibility under different environmental conditions, allowing them to continuously and rapidly adapt to alterations in their environment. A large portion of many plant genomes consists of transposable elements (TEs) that create new genetic variations within plant species. Different types of mutations may be created by TEs in plants. Many TEs can avoid the host's defense mechanisms and survive alterations in transposition activity, internal sequence and target site. Thus, plant genomes are expected to utilize a variety of mechanisms to tolerate TEs that are near or within genes. TEs affect the expression of not only nearby genes but also unlinked inserted genes. TEs can create new promoters, leading to novel expression patterns or alternative coding regions to generate alternate transcripts in plant species. TEs can also provide novel cis-acting regulatory elements that act as enhancers or inserts within original enhancers that are required for transcription. Thus, the regulation of plant gene expression is strongly managed by the insertion of TEs into nearby genes. TEs can also lead to chromatin modifications and thereby affect gene expression in plants. TEs are able to generate new genes and modify existing gene structures by duplicating, mobilizing and recombining gene fragments. They can also facilitate cellular functions by sharing their transposase-coding regions. Hence, TE insertions can not only act as simple mutagens but can also alter the elementary functions of the plant genome. Here, we review recent discoveries concerning the contribution of TEs to gene expression in plant genomes and discuss the different mechanisms by which TEs can affect plant gene expression and reduce host defense mechanisms.
\end{abstract}

Keyword: Transposable elements; Gene expression; Plant development; TEs silencing; Stress conditions 